\title{
Malformación arteriovenosa uterina. A propósito de un caso
}

\author{
Paloma Badía Agustí ${ }^{\text {, Silvia Tamarit Bordes }}{ }^{2}$, Inmaculada Alcover Barrachina ${ }^{3}$, Gemma \\ Tamarit Bordes 4 , Patricia Pérez-Moneo Pérez ${ }^{5}$, María Reyes Balanzá Chancosa. 6 \\ ${ }^{1}$ Médico Residente de Ginecología y Obstetricia, Hospital Doctor Peset. ${ }^{2}$ Médico Adjunto de Ginecología y Obstetricia, \\ Hospital Doctor Peset. ${ }^{3}$ Médico Adjunto de Ginecología y Obstetricia, Hospital Doctor Peset. ${ }^{4}$ Médico Adjunto de \\ Ginecología y Obstetricia, Hospital de Manises. ${ }^{5}$ Médico Residente de Ginecología y Obstetricia, Hospital Doctor Peset. \\ 6 Jefe de Servicio de Ginecología y Obstetricia, Hospital Doctor Peset. Valencia, España.
}

\section{RESUMEN}

Paciente de 37 años que acudió a urgencias por metrorragia, G3P1A2, el último de ellos un aborto tardío en gestación gemelar monocorial biamniótica de 20 semanas hacía dos meses. En la ecografía destaca en zona miometrial un área de marcada vascularización de $67 \times 45 \mathrm{~mm}$. Tras descartar proceso residual gestacional y ante la sospecha de malformación arteriovenosa uterina se solicitó resonancia magnética nuclear y angiotac, tras confirmar la malformación vascular se programó para embolización de ambas arterias uterinas con el fin de preservar la fertilidad.

\section{PALABRAS CLAVES: Malformación arteriovenosa uterina, metrorragia, embolización arterias uterinas, malformación vascular}

\section{SUMMARY}

A 37-year-old patient came to the emergency due to metrorrhagia, G3P1A2, the last of them a late abortion in a 20 weeks monochorionic diamniotic twin pregnancy two months ago. Ultrasound revealed an intramiometrial area of $67 \times 45 \mathrm{~mm}$ with increased vascularization area. A diagnosis of an arteriovenous malformation was considered. In order to confirm the diagnosis pelvic magnetic resonance and angiogram was performed. Once the vascular malformation was confirmed the patient was scheduled for transcatheter arterial embolization in order to preserve fertility.

\section{KEY WORDS: Arteriovenous uterine malformation, metrorrhagia, uterine artery embolization, vascular malformation}

\section{INTRODUCCIÓN}

La malformación arteriovenosa uterina (MAVU), también conocida como fístula arteriovenosa o aneurisma cirsoide es una causa rara de sangrado genital $(1,2)$. A pesar de que no han sido publicados un gran número de casos, se piensa que esta entidad esta subdiagnosticada, ya que puede cursar de forma asintomática e incluso presentar resolución espontánea sin precisar tratamiento (3-7).

El propósito de este caso es recordar la existencia de esta patología, la cual presenta un diagnóstico sencillo mediante ecografía- Doppler. Es importante un diagnóstico precoz de la MAVU, ya que de esta forma es posible iniciar tratamiento médico, y en el caso de precisar tratamiento quirúrgico, poder 
ofertar la posibilidad de la embolización de arterias uterinas con el fin de preservar la fertilidad $(1,6,8)$.

\section{Caso clínico}

Paciente de 37 años que acudió a urgencias por metrorragia de dos semanas de evolución. Sin antecedentes personales de interés. Como antecedentes obstétricos: G3P1A2, el último de ellos un aborto tardío en gestación gemelar monocorial biamniótica de 20 semanas hacía dos meses.

El aborto se diagnosticó en el transcurso de una visita de control obstétrico al que era sometida la paciente, objetivándose un hidrops fetal del primer feto y un stuck twin del segundo feto.

Se realizó aborto medicamentoso con misoprostol vía vaginal sin incidencias destacables y sin requerir aspirado endometrial posterior.

La ecografía de control un mes posterior al aborto, evidenció una línea endometrial fina y regular.

A los dos meses del aborto presentó episodio de metrorragia intensa, visualizándose en la ecografía un útero de 73 x 67 × 50 mm, con línea endometrial homogénea desplazada hacia la derecha de 4,4 mm. En cara posterior y lateral derecha uterina, en zona miometrial, se visualiza área de marcada vascularización de 67 × 45 mm (Figura 1). Ambos ovarios de aspecto normal.

Ante la presencia de sangrado abundante y vascularización intramiometrial abigarrada, y tras descartar enfermedad trofoblástica gestacional (BHCG negativa y AP placenta normal) se inició estudio para descartar malformación arteriovenosa uterina adquirida mediante resonancia magnética $(\mathrm{RM})$ y angioTAC y se pautó tratamiento con Edelsin (norgestimato/etinilestradiol) con control del sangrado (inicialmente $1 \mathrm{c} / 8 \mathrm{~h}$ y posteriormente 1/24h).

La RM pélvica con contraste muestra lesión en cara posterior de cuerpo uterino, de $5 \mathrm{~cm}$ de diámetro máximo, que impronta y desplaza endometrio hacia delante, con múltiples vacíos de señal correspondientes a vasos de alto flujo que tras administración de contraste presentan intenso realce, con drenaje precoz por venas dependientes de ramas hipogástricas. Todo ello corresponde a malformación arteriovenosa miometrial con probable afectación endometrial (Figura 2). Se solicitó angio-TAC por su mayor resolución espacial. Esta muestra en cara posterior del cuerpo uterino una lesión hipervascular que desplaza endometrio hacia delante, con múltiples vasos arteriales de alto flujo, dependientes de ambas arterias uterinas, con drenaje precoz por venas dependientes de ramas hipogástricas. Todo ello estaría en relación con una malformación arteriovenosa miometrial con probable afectación endometrial (Figura 3).

Ante el diagnóstico de MAVU adquirida se programó a la paciente para embolización arterial de forma electiva. La embolización arterial selectiva de ambas arterias uterinas se realizó tres semanas después de completar el estudio por parte del equipo de Radiología Intervencionista mediante infusión de DMSO y embolización con Onyx, con buen resultado morfológico con oclusión del nidus y ramas aferentes (Figura 4). En la ecografía de control tras embolización a las 24 horas, se observó un área sin realce en el centro del útero de $4 \mathrm{~cm}$ de diámetro, que corresponde a la zona de necrosis postembolización. Tras $48 \mathrm{~h}$ de hospitalización la paciente fue dada de alta.

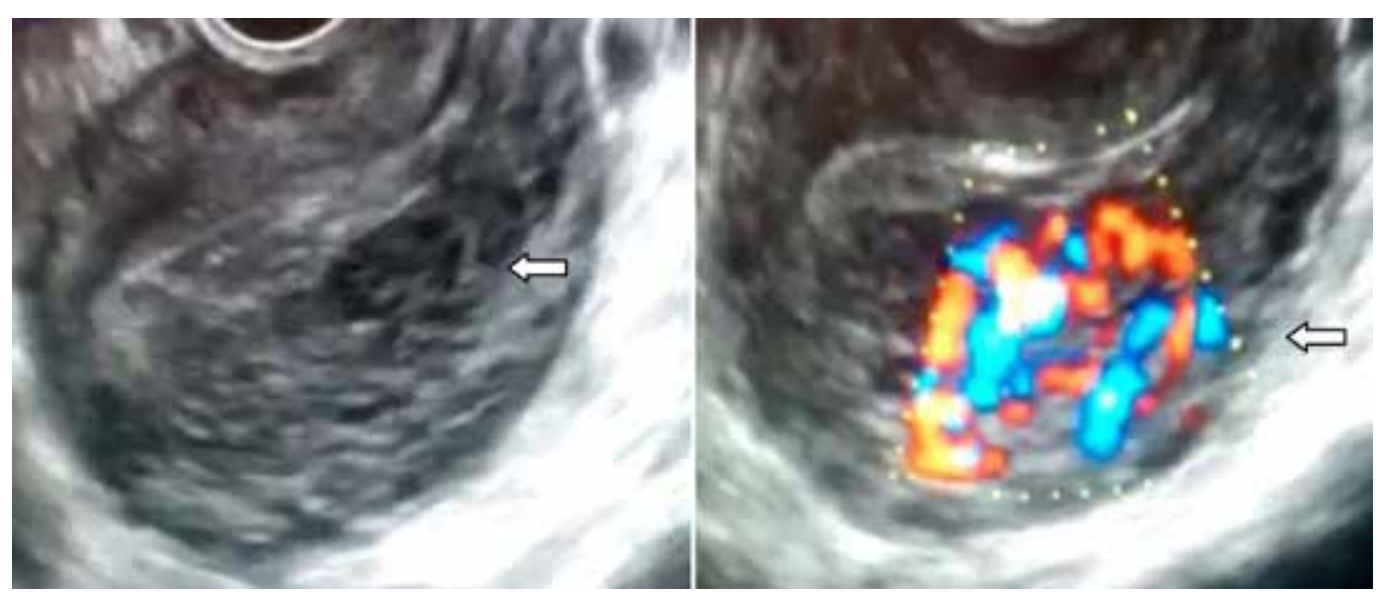

Figura 1. Ecografía transvaginal. Corte en el que se visualiza útero con línea endometrial fina y homogénea. En la cara posterior uterina, en zona miometrial, se visualiza un área de aspecto heterogéneo, con zonas econegativas en su interior de marcada vascularización correspondiente con la malformación arteriovenosa uterina (flecha). 

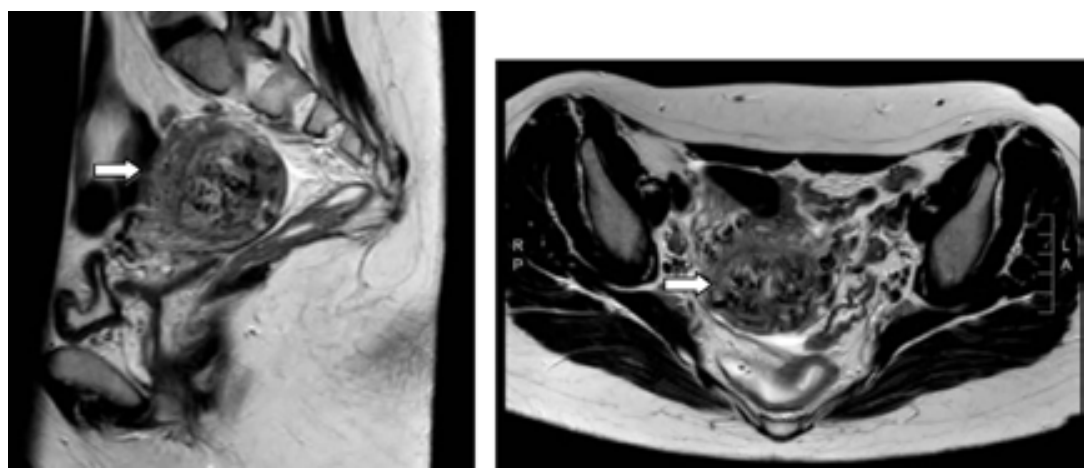

Figura 2. Resonancia magnética pélvica. Se visualiza malformación arteriovenosa miometrial en cara posterior de cuerpo uterino, de $5 \mathrm{~cm}$ de diámetro máximo, que impronta y desplaza endometrio hacia delante, con múltiples vacíos de señal correspondientes a vasos de alto flujo (flechas).

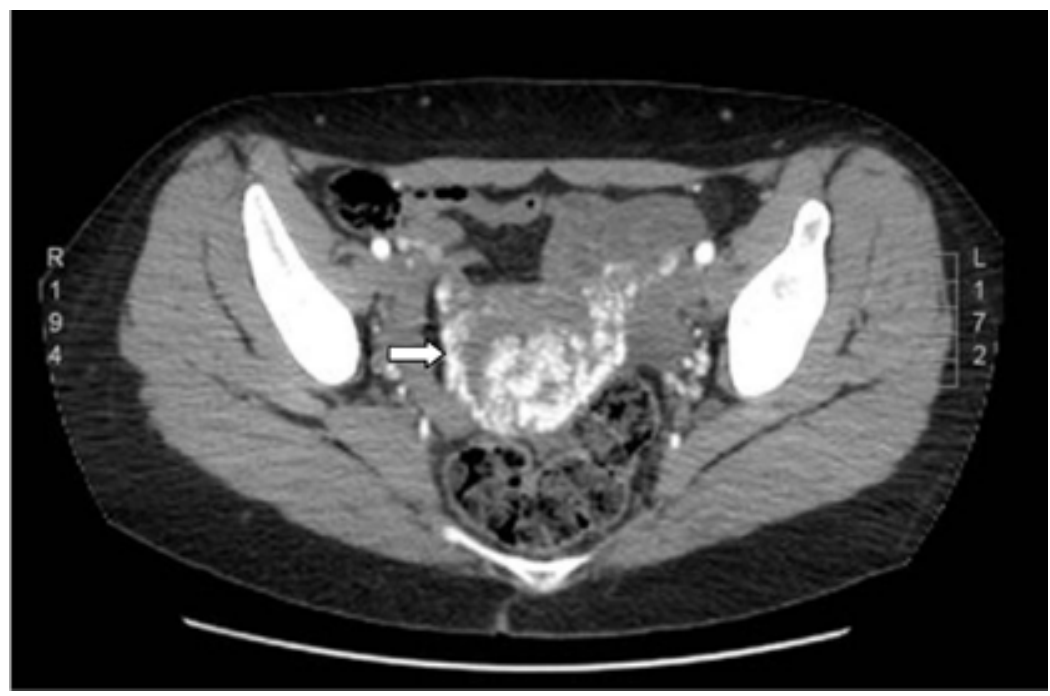

Figura 3. Angio TAC. Malformación arteriovenosa uterina. Lesión hipervascular con múltiples vasos arteriales de alto flujo (flecha), dependientes de ambas arterias uterinas, con drenaje precoz por venas dependientes de ramas hipogástricas.

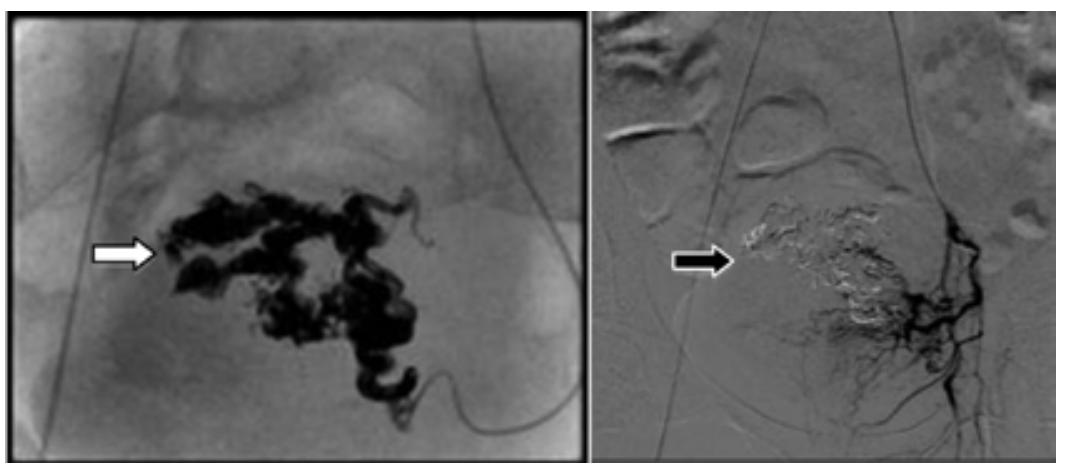

Figura 4. Embolización arterial selectiva. Malformación arteriovenosa previa a la embolización arterial (flecha blanca) y tras la embolización (flecha negra). 
En la visita de control un mes tras la embolización, la paciente presentaba una menstruación normal y en la ecografía se visualizó en cara posterior fúndica a nivel miometrial, imagen con áreas hiperrefringentes de $31 \times 25 \mathrm{~mm}$, no captante de Doppler, correspondiente con MAVU embolizada (Figura 5).

A los dos meses se realizó RM de control, presentando una marcada respuesta terapéutica al procedimiento de embolización, observándose ausencia de relleno de las estructuras vasculares de la malformación arteriovenosa en vertiente posterior del cuerpo y fundus uterino (Figura 6). Endometrio de grosor normal. La paciente fue dada de alta del procedimiento, con controles ginecológicos hasta resolución completa de la imagen ecográfica.
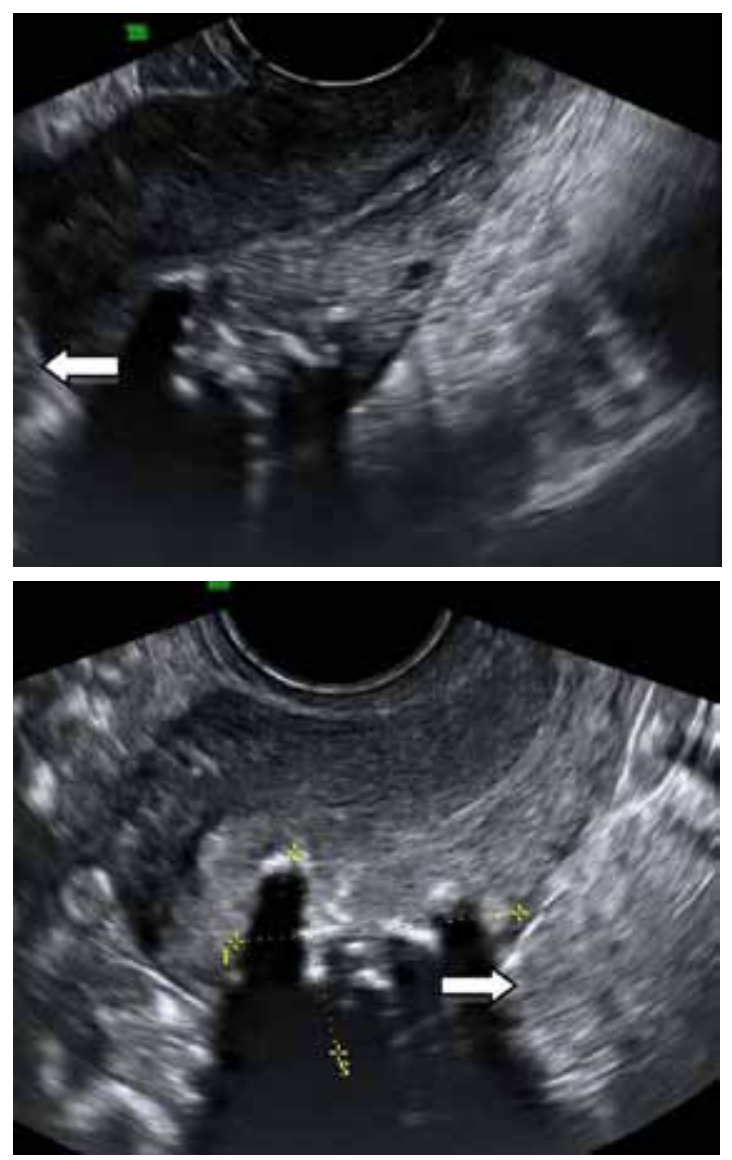

Figura 5. Ecografía transvaginal de control. A nivel de cara posterior uterina, en zona miometrial se visualiza imagen con áreas hiperrefringentes, que no captan Doppler, correspondiente a la malformación arteriovenosa uterina embolizada (flechas).

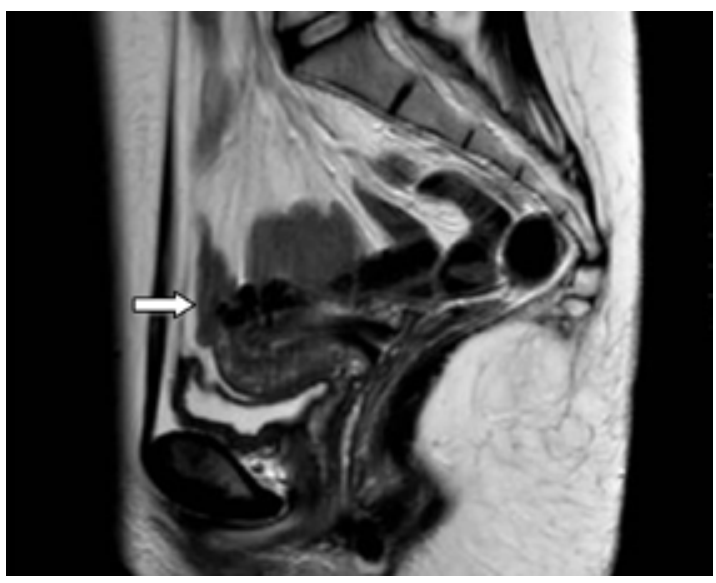

Figura 6. Resonancia magnética de control. Malformación arteriovenosa embolizada en proceso de resolución. Se observa ausencia de relleno de las estructuras vasculares de la malformación arteriovenosa (flecha).

\section{DISCUSIÓN}

La MAVU es un defecto del sistema vascular y esta formada por un conjunto de dilataciones anormales varicosas, arteriales y venosas conectadas, localizadas en el miometrio, sin presencia de capilares interpuestos $(1,4)$. Puede ser congénita o adquirida, siendo esta última la más frecuente. La forma adquirida se presenta de forma típica en mujeres en edad fértil, y presenta múltiples causas o factores de riesgos que predisponen a su aparición, tales como la presencia de un antecedente de neoplasia trofoblástica gestacional, tumor trofoblástico del lecho placentario, antecedentes obstétricos como aborto o gestación múltiple, cirugías uterinas previas como miomectomía, legrado o cesárea, e infecciones, así como la asociación a dietil-etil-bestrol, entre otras $(1,2,4,5,9)$.

La clínica derivada de esta patología es muy diversa, pudiendo permanecer silente en un número variable de casos. En la mayoría de los casos sintomáticos se presenta como una alteración del patrón de sangrado (goteo persistente, metrorragia) o bien puede presentarse como hemorragia masiva con riesgo vital $(1-3,5,6,8)$.

Ante la presencia de un sangrado genital anómalo es necesario la realización de una ecografíaDoppler vía transvaginal, la cual constituye una herramienta suficiente para diagnosticar la malformación (3-5,7-9). En la ecografía se observa de forma típica un miometrio de aspecto heterogéneo, 
con zonas anecoicas irregulares en su interior que pueden confundir con zona de adenomiosis. Al aplicar Doppler-color, en las zonas anecoicas, se visualiza el ovillo vascular, con flujo multidireccional, de alta velocidad y baja resistencia, que producen imagen de mosaico vascular. Este ovillo se presenta en el $95 \%$ de los casos de las formas adquiridas $(1,2,7,9)$.

Ante la sospecha de una MAVU, se debe solicitar la medición de BHCG para descartar proceso gestacional residual (aborto, neoplasia trofoblástica gestacional o tumor trofoblástico del lecho placentario). Para completar el diagnóstico se solicitará RMN o TAC, siempre que este disponible, sin embargo el método diagnóstico definitivo, que posee la mayor sensibilidad y especificidad es la angiografía $(1,2,4,9)$.

El tratamiento varía en función de la clínica y de la estabilidad hemodinámica de la paciente, desde manejo expectante hasta histerectomía, sin embargo el tratamiento más eficaz es la embolización de las arterias uterinas, cuya principal ventaja frente a la histerectomía es la preservación de la fertilidad $(1,3,4,7,9,10)$. Es importante recordar que el legrado está contraindicado ya que suele agravar el sangrado pudiendo causar la muerte $(2,5,6)$. En nuestro caso la paciente deseaba un nuevo embarazo y debido al diagnóstico precoz, fue posible realizar la embolización de forma programada satisfactoriamente.

\section{REFERENCIAS}

1. Machado L, Raga F, Chagas K, Bonilla F, Castillo JC, Bonilla-Musoles F. La malformación arteriovenosa uterina. Una lesión más frecuente y grave de lo sospechado. Prog Obstet Ginecol 2010;53(1):10-7.
2. Rodríguez A, Escartín I, Riazuelo G, Zaragozano R. Malformación arteriovenosa uterina como rara causa de sangrado genital. Radiología 2002;44(2):81-3.

3. Sellers F, Palacios-Marqués A, Moliner B, Bernabeu $R$. Uterine arteriovenous malformation. BMJ Case Reports 2013. pii: bcr2012008443. doi: $10.1136 / \mathrm{bcr}-$ 2012-008443.

4. Marcos Lapera C, Barrachina Tortajada R, Casanova Fuset A, Canet Esteve MA, Ferrer Puchol MD, Naranjo La Puerta F. Malformación arteriovenosa uterina. Una causa poco frecuente de metrorragia. Revisión de la literatura y presentación de un caso. Fertilidad 2010; 27(4):307-11.

5. Lacunza Paredes RO. Malformación arteriovenosa uterina, como causa poco frecuente de hemorragia uterina anormal. Rev Per Ginecol Obstet 2013;59 (3):203-5.

6. Molvi S, Dash K, Rastogi H, Khanna S. Transcatheter embolization of uterine arteriovenous malformation: report of 2 cases and review of literature. J Minim Invasive Gynecol 2011;18(6):812-9.

7. Timmerman D, Van den Bosch T, Peeraer K, Debrouwere E, Van Schoubroeck D, Stocks L. Vascular malformations in the uterus: ultrasonographic diagnosis and conservative management. Eur J Obstet Gynecol Reprod Biol 2000;92(1):171-8.

8. Hasbun J, Palavecino P, Segura P, Chávez I, Burgos $\mathrm{N}$, Tobar $\mathrm{H}$, Aliaga A. Malformación arterio-venosa uterina y hemorragia obstétrica: diagnóstico y manejo en tres casos clínicos. Rev Chil Obstet Ginecol 2014;79(3):199-208.

9. O'Brien P, Neyastani A, Buckley AR, Chang SD, Legiehn GM. Uterine arteriovenous malformations. From diagnosis to treatment. J Ultrasound Med 2006;25(11):1387-92

10. Wang Z, Chen J, Shi H, Zhou K, Sun H, Li, Pan J, Zhang X, Liu W, Yang N, Jin Z. Efficacy and safety of embolization in iatrogenic traumatic uterine vascular malformations. Clin Radiol 2012;67(6):541-5. 